\title{
Interactions in Chaotic Nanoparticles: Fluctuations in Coulomb Blockade Peak Spacings
}

\author{
Denis Ullmo ${ }^{1}$ and Harold U. Baranger ${ }^{2}$ \\ ${ }^{1}$ Laboratoire de Physique Théorique et Modèles Statistiques (LPTMS), 91405 Orsay Cedex, France \\ ${ }^{2}$ Department of Physics, Duke University, Box 90305, Durham NC 27r08-0305, USA
}

(March 1, 2001)

\begin{abstract}
We use random matrix models to investigate the ground state energy of electrons confined to a nanoparticle. Our expression for the energy includes the charging effect, the single-particle energies, and the residual screened interactions treated in Hartree-Fock. This model is applicable to chaotic quantum dots or nanoparticles - in these systems the single-particle statistics follows random matrix theory at energy scales less than the Thouless energy. We find the distribution of Coulomb blockade peak spacings first for a large dot in which the residual interactions can be taken constant: the spacing fluctuations are of order the mean level separation $\Delta$. Corrections to this limit are studied using the small parameter $1 / k_{F} L$ : both the residual interactions and the effect of the changing confinement on the single-particle levels produce fluctuations of order $\Delta / \sqrt{k_{F} L}$. The distributions we find are significantly more like the experimental results than the simple constant interaction model.
\end{abstract}

PACS numbers: 73.23.Hk, 05.45.Mt, 71.10.Ca, 73.20.Dx

\section{INTRODUCTION}

The ground state properties of electrons in nanoparticles or quantum dots - finite systems of fermions interacting via Coulomb forces - remain incompletely understood. One valuable way to probe these systems is via electrical transport (for reviews see Refs. 11- 1 ). The dominant effect is the suppression of the conductance $G$ because of the charging energy needed to add an electron to the nanoparticle and so allow current to flow. This Coulomb blockade $(C B)$ is an essentially classical effect, and the charging energy is simply $E_{C}=e^{2} / 2 C$ where $C$ is the capacitance of the nanoparticle. The blockade can be lifted by adjusting the potential on the particle - with a gate voltage $V_{g}$, for instance - so that the energy for $N$ electrons is the same as that for $N+1$. The conductance through the nanoparticle has peaks at these degeneracy points; in fact, $G\left(V_{q}\right)$ is periodic with a spacing of $e^{2} / C$ between the peaks [1.2].

At low temperature the electron remains coherent in the dot and so quantum interference effects must be included in the Coulomb blockade [5 8]. Two main effects occur: (1) the peak heights fluctuate from peak to peak because of varying coupling between the wavefunction in the nanoparticle and the leads, and (2) the spacings between the peaks fluctuate because of interference contributions to the ground state energy. Peak heights have been studied first theoretically [9] and then experimentally [19 21]; agreement is good once effects of temperature [13],19] and classical dynamics 16 18] are taken into account.

In this paper we focus on quantum effects in the peak spacings. These are directly related to the interference contributions to the ground state energy. One obvious contribution is the single-particle energy levels caused by the confinement in the quantum dot. But there may also be contributions coming from the interactions in the dot. We will show that for typical parameters characterizing experimental quantum dots or nanoparticles the contributions coming from the interactions must be included.

The simplest treatment of Coulomb blockade peak spacing fluctuations results from writing the ground state energy as the sum of the classical electrostatic energy and the energies of the occupied single particle states [5]. Such an expression for the ground state energy of a many Fermion system has a long and venerable history in atomic and nuclear physics 222 24. Using $\mathcal{E}$ for the energy in the presence of the gate voltage while reserving $E$ for $V_{g}=0$, we have for $N$ particles

$$
\begin{aligned}
\mathcal{E}_{N}\left(V_{g}\right) & =E_{N}-\left(C_{g} / C\right) e N V_{g} \\
E_{N} & =\left(e^{2} / 2 C\right) N^{2}+\sum_{\text {occupied } i \sigma} \epsilon_{i \sigma}
\end{aligned}
$$

where $C_{g}$ is the capacitance of the gate to the dot and $\left\{\epsilon_{i \sigma}\right\}$ are the single-particle energy levels. This treatment, known as the constant-interaction model (CI), is still an important point of reference in the field. The gate voltage $V_{g}^{*}$ at which a peak in the conductance occurs is given by the condition $\mathcal{E}_{N}\left(V_{g}^{*}\right)=\mathcal{E}_{N+1}\left(V_{g}^{*}\right)$. The spacing between two peaks is, then, proportional to the (discrete) inverse compressibility $\Lambda$

$$
\begin{aligned}
\left(V_{g}^{*}\right)_{N \rightarrow N+1}-\left(V_{g}^{*}\right)_{N-1 \rightarrow N} \propto \\
E_{N+1}+E_{N-1}-2 E_{N} \equiv \Lambda_{N} .
\end{aligned}
$$

In the case of the CI model, one gets the simple prediction 25]

$$
\begin{array}{ll}
\Lambda_{N}=e^{2} / C & \text { for odd } N \\
\Lambda_{N}=e^{2} / C+\left(\epsilon_{N / 2+1}-\epsilon_{N / 2}\right) & \text { for even } N .
\end{array}
$$


Note the drastic odd/even effect in the quantum correction to the classical result $e^{2} / C$ because of the spin of the electron.

A number of experiments have studied the distribution of CB peak spacings in semiconductor quantum dots 25 29, a particularly flexible and convenient type of nanoparticle. The overall result has been striking disagreement with the CI model. The main discrepancy is that there is no sign of the strong odd/even effect predicted by Eq. (3), though there is evidence of a weak odd/even effect 29,30]. In addition, in some of the experiments the widths of the observed distributions are significantly larger than what is predicted using the CI model and assuming the single-particle energy levels $\epsilon_{i}$ are distributed according to the appropriate Gaussian random matrix ensemble. These discrepancies indicate unambiguously that interaction effects play a significant role in the fluctuating part of the Coulomb Blockade peak position.

Theoretically accounting for this disagreement is difficult because of two intrinsic characteristics of the problem: (1) the fairly large number of electrons contained in the dot (typically $N \sim 100-1000$ ), and (2) the long range character of the Coulomb interaction which requires that screening has to be correctly taken into account. The first of these points makes it delicate to extend to realistic dots the results obtained by exact multiparticle diagonalization [25.31, which can only treat problems with a few electrons $(\lesssim 15)$. To reach the experimental number of particles in a numerical approach, one has to rely on some self-consistent technique. One approach is Hartree-Fock which has been recently used in quantum dots [32 35]. Then, however, the second point comes into play. Indeed, screening is a higher-order interaction effect, and it is well known for the Coulomb interaction that the exchange terms can be significantly over evaluated in selfconsistent Hartree-Fock (for the bulk see Ref. [36] but this applies also for restricted geometries). This makes it rather difficult to interpret numerical self-consistent Hartree-Fock calculations using the bare Coulomb interaction as input. Another possible self-consistent approach is, of course, density functional theory in the local density or local spin density approximation 37 39. Here the screening is properly handled but approximations are made in the exchange term. Since, as we explain in detail in this paper, the subtle interplay between exchange, residual direct interactions, and single-particle level fluctuations is critical in describing quantum dot physics, results from this method are also difficult to generalize.

An alternative theoretical approach is to formally carry out an RPA screening calculation and then use a shortrange screened interaction in the subsequent evaluation of the magnitude of various effects 40 42, 8]. The nature of the screened interaction used is, then, important. Since experimentally the system size is much larger than the screening length, the most natural choice for the in- teraction is the bulk RPA screened interaction. For momentum smaller than $2 k_{F}$ this is correctly approximated by the Thomas-Fermi expression. Throughout this paper we consider two dimensional systems, in which case

$$
\begin{aligned}
V_{\mathrm{TF}}(\mathbf{r}) & =\int \frac{d \mathbf{q}}{(2 \pi)^{2}} \hat{V}_{\mathrm{TF}}(\mathbf{q}) \exp [i \mathbf{q} \cdot \mathbf{r}] \\
\hat{V}_{\mathrm{TF}}(\mathbf{q}) & =\frac{\nu^{-1}}{1+r_{s}^{-1}\left(|\mathbf{q}| / k_{F}\right) / \sqrt{2}}
\end{aligned}
$$

where $\nu$ is the mean density of states (including spin degeneracy), and $r_{s}=r_{0} / a_{0}$ with $\pi r_{0}^{2}$ the average area per electron and $a_{0}$ the Bohr radius in the semiconductor. It should be born in mind that although $\hat{V}_{\mathrm{TF}}$ is proportional to $r_{s}$ for small $r_{s}$, it becomes largely independent of $r_{s}$ when $r_{s} \gtrsim 1$, which is the case experimentally. In fact, within any RPA-like approach the interaction cannot become stronger than

$$
V_{\text {zero-range }}(\mathbf{r})=\nu^{-1} \delta(\mathbf{r})
$$

which we refer to as the zero-range limit of the potential. Once screening is included, the problem is that the interaction is small, in a sense that we shall make more explicit below, even in the strongest zero-range limit. Thus the self-consistent approach should give roughly the same answer as the CI model with first-order perturbation in the screened interaction added. This is exactly the picture one has of weakly interacting Landau quasi-particles, with the obvious drawback that it is not straightforward to extract large contributions using this approach (see e.g. Refs. 40 42]).

In order to obtain a large interaction effect, one possibility is to give up the Fermi-liquid-like approach and to assume, for instance, that for some reason screening is not as efficient in a nanoparticle as in the bulk, and so conclude that large zero-range interactions, not amenable to a perturbative treatment, should be used 25, 31, 40,41. Before taking this drastic step, it seems reasonable, however, to see how far one can go within the more standard Fermi liquid description. Indeed, even in this framework, we know that the CI model is inadequate.

An accurate treatment of the spacing or inverse compressibility certainly requires that the average effect of the residual interactions be added to the CI model. By "residual interactions" we mean interactions beyond the simple classical effect taken into account in the constant interaction model and, in particular, interactions between the quasiparticles after the bare electrons are dressed through screening. While they make a smaller contribution to the total energy than those considered in Eq. (1), residual interactions make a contribution of order the mean level separation to the second difference $\Lambda$, comparable to the single-particle contribution in Eq. (3) 40, 42, 46]. In the limit of a large nanoparticle one whose typical dimension $L$ is many times the electron 
wavelength, $k_{F} L \gg 1$ - only the average effect of residual interactions needs to be added to the CI model 42 45. We shall below refer to this approximation as the constant exchange model. The intuitive argument for this is that the effect of residual interactions involves integrating wavefunctions over the entire volume of the nanoparticle and so self-averages: the particular characteristics of any given wavefunction are simply integrated out. Even if one neglects the fluctuations of the residual interaction terms, because of fluctuations of the single-particle levels, the "constant exchange" contribution can modify the total spin of the nanoparticle by favoring non-trivial occupation of the single-particle orbitals and, as a consequence, will significantly modify the fluctuations of the peak spacings 40,42. The ground state spin of a large nanoparticle in this regime has been examined in detail $43,45,47$, but curiously the corresponding distribution of Coulomb blockade peak spacings has not appeared explicitly (see however Ref. [42]). Therefore, before turning to effects caused by fluctuations in the interactions - our main interest in this paper - we present below the distribution in the constant exchange limit $\left(k_{F} L \gg 1\right)$ to use as a reference point.

In the experiments, however, $k_{F} L$ is not very large because of the need to have the mean level separation larger than the temperature. This suggests that the variations in interactions caused by properties of individual wavefunctions are important. The main goal of this paper is, therefore, to study the effect of fluctuations in the electron-electron interactions on the distribution of $\mathrm{CB}$ peak spacings.

We limit our study here to the case of chaotic dynam$i c s$ within the dot, for which a random matrix description of the non-interacting limit can be used 48. Much as in the case of diffusive transport, completely chaotic dynamics introduces an important additional energy scale (and only one): the Thouless energy, $E_{\mathrm{Th}}=\hbar / \tau$, is the inverse of the transit time across the dot. It is generally larger than the mean level separation $\Delta$ but smaller than the Fermi energy; we will assume $\Delta \ll E_{\mathrm{Th}} \ll E_{F}$. On energy scales less than $E_{\mathrm{Th}}$, a universal random matrix description holds while for larger energies (shorter times) the individual dynamics of the system comes into play. We will see that taking account of this additional energy scale is critical in describing CB peak spacings in nanoparticles.

We focus on two effects in particular: (1) the fluctuation in the residual Hartree and Fock contributions to the ground state energy and (2) the change in the singleparticle energies because of changes in the mean field potential as electrons are added to the dot [42], an effect we refer to as "scrambling". For two dimensional quantum dots, the changes in energy are evaluated to leading order in the smallness of the dot, the constant exchange model being used as the starting and reference point. We find that both effects contribute terms which are of order $\Delta / \sqrt{k_{F} L}$, up to logarithmic corrections. The resulting distribution of CB peak spacings looks more like the experiment - it has a more Gaussian and symmetric form, for instance - but still deviates from the measurements in significant ways.

The paper is organized as follows. Our starting point is a semiclassical expansion of the ground state energy described in Section III an expansion in $1 / k_{F} L$. The important large dot limit is presented in Section III. The next two sections discuss the two main issuesfluctuations of the residual interaction contributions (Section IV) and scrambling (Section V). Section VI takes a first step beyond the Gaussian model introduced in Section IV. Finally, our conclusions, including a discussion of the experimental results, appear in Section VII.

\section{APPROACH: SEMICLASSICAL CORRECTIONS}

In the Coulomb blockade through quantum dots we are faced with a classical theory which works remarkably well to which we want to add quantum effects to leading order. The small parameter is the standard semiclassical one, $\hbar$. In our context, this is equivalent to an expansion in $1 / k_{F} L$ where $k_{F}$ is the Fermi wavevector and $L$ is the typical size of the nanoparticle. Another useful way to view the corrections is in terms of the dimensionless conductance, $g$, given as the ratio of the transit rate $\hbar v_{F} / L$ to the mean level separation $\Delta$; in this case the expansion is in terms of $1 / g$, as in the diffusive mesoscopic regime 49. For 2-dimensional systems, such as the ones we consider here, these two parameters are proportional $\left(g=k_{F} L / 2 \pi\right)$.

We proceed by using the method of V. M. Strutinsky 50,51 in which the dependence of many-body ground state quantities on particle number can be decomposed into an average and a fluctuating part. While the average part varies smoothly with particle number, the fluctuating part reflects the shell structure of the system. The smooth part is the bulk energy per unit volume integrated over the finite-size system, and the oscillating contributions come from quantum interference effects explicitly caused by the confinement. By supposing that the smooth part is known while the unknown oscillatory contribution is a correction, Strutinsky introduced a physically motivated systematic approach to obtain the shell corrections 50,51. This shell correction method is essentially a semiclassical approximation. It rests on the fact that the number of particles in the system considered is large, rather than on the interaction between the particles being weak. (One must, of course, work in a regime where the smooth starting point is basically valid.) Since the quantum dots in which we are interested contain on the order of 100 electrons, they are a perfect place to 
apply the Strutinsky method. We use the formulation recently developed specifically for quantum dots in Ref. 52 .

Density functional theory guarantees that the ground state energy of a nanoparticle can be written as a functional of its density, $E_{\mathrm{DFT}}=\mathcal{F}_{\mathrm{DFT}}\left[n_{\mathrm{DFT}}\right]$. Neglecting quantum interference effects in this functional corresponds to a generalized Thomas-Fermi approximation (generalized because bulk local exchange and correlation can be included) which can also be written as a functional of a (smooth) density, $E_{\mathrm{GTF}}=\mathcal{F}_{\mathrm{GTF}}\left[n_{\mathrm{GTF}}\right]$. The quantum interference part of the energy, $E_{\mathrm{DFT}}-E_{\mathrm{GTF}}$, can be obtained approximately by expanding $n_{\mathrm{DFT}}$ about $n_{\mathrm{GTF}}$ and solving the resulting density functional equations order by order in the oscillating part of the density $n_{\mathrm{DFT}}-n_{\mathrm{GTF}}$. To carry out this procedure an explicit form for the exchange-correlation functional is required. Based on the local density approximation, the result derived in Ref. 52 is

$$
E_{\mathrm{DFT}} \simeq E_{\mathrm{GTF}}+\Delta E^{(1)}+\Delta E^{(2)}
$$

where the first and second order correction terms are

$$
\begin{aligned}
& \Delta E^{(1)}=\mathcal{E}_{1 \mathrm{p}}^{\mathrm{osc}}\left[V_{\mathrm{eff}}\left[n_{\mathrm{GTF}}\right]\right] \equiv \sum_{i=1}^{N} \delta \epsilon_{i} \\
& \Delta E^{(2)}=\frac{1}{2} \int d \mathbf{r} d \mathbf{r}^{\prime} \tilde{n}^{\mathrm{osc}}(\mathbf{r}) V_{\mathrm{sc}}\left(\mathbf{r}, \mathbf{r}^{\prime}\right) \tilde{n}^{\mathrm{osc}}\left(\mathbf{r}^{\prime}\right) .
\end{aligned}
$$

The first-order correction is the oscillatory part of the single-particle energy, $\mathcal{E}_{1 \mathrm{p}}^{\text {osc }}$, calculated in the smooth Thomas-Fermi potential, $V_{\mathrm{eff}}\left[n_{\mathrm{GTF}}\right]$; that is, it is the sum of the deviations $\delta \epsilon_{i}$ of the single-particle levels $\epsilon_{i}$ from their mean values. In the second-order correction, $\tilde{n}^{\text {osc }}$ is the deviation of the quantum mechanical density calculated in the Thomas-Fermi potential from the smooth starting point, $\tilde{n}^{\text {osc }} \equiv n\left[V_{\text {eff }}\left[n_{\mathrm{GTF}}\right]\right](\mathbf{r})-n_{\mathrm{GTF}}$, and $V_{\mathrm{sc}}\left(\mathbf{r}, \mathbf{r}^{\prime}\right)$ is the screened interaction in the nanoparticle. To the order that we are working, using $\tilde{n}^{\text {osc }}$ here is equivalent to using $n_{\mathrm{DFT}}-n_{\mathrm{GTF}}$ [52].

In this approach, the ground state energy is, then, the sum of a classical contribution - the generalized-ThomasFermi result $E_{\mathrm{GTF}}$ - and two quantum contributions$\Delta E^{(1)}$ and $\Delta E^{(2)}$. With the inclusion of only the firstorder contribution, Eq. (6) is simply the CI model for the ground state energy. Because we have the corrections to this model, we can see exactly when it is applicable. The second-order correction, Eq. (8), has a natural interpretation: the ripples in the density caused by interference (the Friedel oscillations in this setting) interact with each other via an interaction screened by the smooth Thomas-Fermi fluid already present. In fact, the bulk Thomas-Fermi interaction Eq. (4) can often be used for $V_{\text {sc }}$. Taking the $r_{s} \rightarrow \infty$ limit formally yields the zerorange interaction limit (5). This is equivalent to using a Hubbard Hamiltonian on a square lattice with hopping term $t$ and interaction $U$ with $U / t=\pi$.
The result Eq. (6) is, however, inadequate for our purposes here because of the local density approximation used. In particular, the spin degree of freedom is handled poorly in this approximation: while Eq. (8) gives the "direct" interaction between the density ripples, an analogous "exchange" term is missed. A better treatment of the interactions in density functional theory could presumably yield this additional term, by including offdiagonal terms in the density matrix, for instance. In fact, an RPA treatment of the interaction in the diffusive case yields an energy with exactly this structure 42, 8. Motivated by these physical considerations, we simply add the exchange term by hand to the expression for the energy $E_{\mathrm{DFT}}$.

For the purposes of deriving CB peak spacing distributions, we thus consider 3 contributions to the ground state energy: (1) the Thomas-Fermi energy, (2) the deviation of the single-particle energy from its mean, and (3) a Hartree-Fock like treatment of the residual screened interaction. Without the residual interactions, a $N$ particle state is, of course, a Slater determinant in the basis of single-particle eigenstates $\psi_{i}(\mathbf{r})$; it is characterized by the occupation numbers $\left(n_{0 \sigma}, n_{1 \sigma}, \ldots\right)$ where $n_{i \sigma}=0$ or $1, \sigma=+1$ or -1 labels the spin degree of freedom, and $\sum n_{i \sigma}=N$. In this occupation number representation, the expression for the energy is, then,

$$
\begin{aligned}
\mathcal{E}_{\mathrm{tot}}\left(\left\{n_{i \sigma}\right\}\right) & =E_{\mathrm{GTF}}+\sum_{i \sigma} n_{i \sigma} \delta \epsilon_{i} \\
+ & \frac{1}{2} \sum_{i \sigma, j \sigma^{\prime}} n_{i \sigma} M_{i j} n_{j \sigma^{\prime}}-\frac{1}{2} \sum_{i, j ; \sigma} n_{i \sigma} N_{i j} n_{j \sigma}
\end{aligned}
$$

where

$$
\begin{aligned}
M_{i j} & \equiv \int d \mathbf{r} d \mathbf{r}^{\prime}\left|\psi_{i}(\mathbf{r})\right|^{2} V_{\mathrm{TF}}\left(\mathbf{r}-\mathbf{r}^{\prime}\right)\left|\psi_{j}\left(\mathbf{r}^{\prime}\right)\right|^{2} \\
N_{i j} & \equiv \int d \mathbf{r} d \mathbf{r}^{\prime} \psi_{i}(\mathbf{r}) \psi_{j}^{*}(\mathbf{r}) V_{\mathrm{TF}}\left(\mathbf{r}-\mathbf{r}^{\prime}\right) \psi_{j}\left(\mathbf{r}^{\prime}\right) \psi_{i}^{*}\left(\mathbf{r}^{\prime}\right)
\end{aligned}
$$

are the direct and exchange contributions, respectively. This is the starting point for our study of CB peak spacings.

In order to derive the statistical properties of the CB peak spacings from Eq. (9), the statistical properties of the single-particle eigenvalues and eigenfunctions must be known. For this purpose we assume that the single-particle classical dynamics in the nanoparticle is completely chaotic. In this case it is well-known that the single-particle quantum mechanics is accurately described by random matrix theory (RMT) on an energy scale smaller than the Thouless energy $E_{\mathrm{Th}}$ (which, again, is the inverse time of flight across the system) 48,53 . We consider only the case of no symmetry here, so that the energy levels $\left\{\epsilon_{i}\right\}$ obey Gaussian Unitary Ensemble (GUE) statistics. Because of the spatial integrals in the expressions for $M_{i j}$ and $N_{i j}$, the correlations of the 
wavefunctions are also needed. For the classical Gaussian ensembles, there is no spatial correlation for a given wavefunction, except for that arising from the normalization. This is a direct consequence of the fact that no basis should play a particular role for these matrix ensembles. Here, however, because of the particular role played by the kinetic energy, the plane wave basis is special. More precisely, we should implement that each eigenfunction is localized in this basis on the energy scale $E_{\mathrm{Th}}$. We shall therefore build our random matrix ensemble with the following requirements (a practical implementation is given in section $\mathrm{VI}$ ):

i) On energy scales smaller than the Thouless energy $E_{\mathrm{Th}}$, one should recover standard GUE properties.

ii) On scales larger than $E_{\mathrm{Th}}$, an eigenstate of energy $\epsilon$ should appear localized on the energy surface $|\mathbf{k}|=\sqrt{2 m \epsilon} / \hbar$. More precisely, we require that in a plane wave basis an eigenstate has an envelope of width

$$
\delta k=1 / L
$$

where $L$ is the typical size of the system.

iii) Finally, we have to implement that the fluctuations of energy levels, and also importantly of local density, saturate at the Thouless energy. That is, if one considers a quantity $A$ expressed as a sum over the energy levels in an energy window $\delta E$ about the Fermi energy, as the window widens the fluctuations of $A$ increase until $\delta E \approx E_{\mathrm{Th}}$ beyond which point the fluctuations of $A$ do not further increase.

Except for the fact that the Fermi surface is given an explicit width $\approx E_{\text {Th }}$ due to the finite size $L$ of the system, items i) and ii) are very similar to Berry's modeling of chaotic wave functions 54 which assumes that a random superposition of plane waves describes the correlation on scales smaller than the size of the system $L$. Item iii) is motivated by the known saturation of, for instance, fluctuations in the density of states of chaotic systems at an energy scale of order $\hbar$ divided by the period of the shortest periodic orbit 48]. Note that these requirements will yield a random matrix approach noticeably different from the ones discussed in Refs. 40,43,46.

In this model, the wave function statistics that we find are as follows: for a two dimensional dot of area $A$ (the case that we consider throughout this paper)

$$
A\left\langle\psi_{i}(\mathbf{r}) \psi_{j}^{*}\left(\mathbf{r}^{\prime}\right)\right\rangle=\delta_{i j} J_{0}\left(k_{F}\left|\mathbf{r}-\mathbf{r}^{\prime}\right|\right)
$$

for $\left|\mathbf{r}-\mathbf{r}^{\prime}\right| \ll L$. (In a maximum entropy approach semiclassically restricted by the classical dynamics, this corresponds to keeping only the direct trajectory between $\mathbf{r}$ and $\mathbf{r}^{\prime}$; the approach can be generalized to keep more classical paths [55,18].) In addition, it can be shown that within our random matrix modeling, one has, up to $1 /\left(k_{F} L\right)$ corrections

$$
\begin{aligned}
& \left\langle\psi_{i}\left(\mathbf{r}_{1}\right) \psi_{i}^{*}\left(\mathbf{r}_{2}\right) \psi_{i}\left(\mathbf{r}_{3}\right) \psi_{i}^{*}\left(\mathbf{r}_{4}\right)\right\rangle= \\
& \quad\left\langle\psi_{i}\left(\mathbf{r}_{1}\right) \psi_{i}^{*}\left(\mathbf{r}_{2}\right)\right\rangle\left\langle\psi_{i}\left(\mathbf{r}_{3}\right) \psi_{i}^{*}\left(\mathbf{r}_{4}\right)\right\rangle \\
& \quad+\left\langle\psi_{i}\left(\mathbf{r}_{1}\right) \psi_{i}^{*}\left(\mathbf{r}_{4}\right)\right\rangle\left\langle\psi_{i}\left(\mathbf{r}_{3}\right) \psi_{i}^{*}\left(\mathbf{r}_{2}\right)\right\rangle
\end{aligned}
$$

Alternatively, these statistics can be obtained directly by assuming that the distribution of wavefunctions is Gaussian [55], though in this case special care must be taken with regard to the normalization constraint.

There are alternate routes to our very natural starting point Eq. (9). One that has been developed recently is the "universal Hamiltonian" approach [56, 57, 45, 8] which uses RPA to treat the interactions, RMT properties of the single-particle wavefunctions, and the small parameter $1 / g$ to arrive at an effective Hamiltonian. Treating this effective Hamiltonian in the Hartree-Fock approximation leads to the same expression for the ground state energy that we give above.

We end this section by giving explicit expressions for the inverse compressibility, which is proportional to the peak spacing [Eq. (2)], in two simple cases in which the standard up-down filling of the states is assumed. First consider the case of $N$ even with $n=N / 2$. For the standard filling, it is a singlet state and the two neighboring states for $N-1$ and $N+1$ electrons are doublets. The peak spacing is then

$$
\begin{aligned}
\Delta^{2} E_{N} \text { even } & =\Delta^{2} E_{\mathrm{GTF}}+\delta \epsilon_{n+1}-\delta \epsilon_{n} \\
+ & \sum_{i=1}^{n}\left[2\left(M_{n+1, i}-M_{n, i}\right)-\left(N_{n+1, i}-N_{n, i}\right)\right]
\end{aligned}
$$

On the other hand if $N$ is odd, still assuming the simplest up-down filling, the peak spacing is

$$
\Delta^{2} E_{N+1 \text { odd }}=\Delta^{2} E_{\mathrm{GTF}}+M_{n+1, n+1} .
$$

The second difference of $E_{\mathrm{GTF}}$ is almost equal to the charging energy $e^{2} / C$ but there is a small correction from the residual interactions; this is discussed in detail below.

\section{LARGE DOT LIMIT}

The main simplifying feature in the large dot limit, $k_{F} L \gg 1$, is that the fluctuations of the interactions can be neglected, much as for diffusive nanoparticles 5659 in the large $g$ limit. The distribution of the spacings is determined, then, by the fluctuations of the singleparticle levels and their interplay with the mean residual interactions. Of course, as has been studied previously [40,43 45], the ground state of the nanoparticle does not necessarily follow the simple filling used in Eqs. (15)(16), and this effect must be included in finding the distribution. 
Let us consider the mean and variance of the $M_{i j}$ and $N_{i j}$ for levels near the Fermi level. We have, for instance, using Eq. (15)

$$
\begin{aligned}
\left\langle M_{i j}\right\rangle= & \int d \mathbf{r} d \mathbf{r}^{\prime}\left\langle\left|\psi_{i}(\mathbf{r})\right|^{2}\right\rangle V_{\mathrm{TF}}\left(\mathbf{r}-\mathbf{r}^{\prime}\right)\left\langle\left|\psi_{j}\left(\mathbf{r}^{\prime}\right)\right|^{2}\right\rangle \\
& +\delta_{i j} \int d \mathbf{r} d \mathbf{r}^{\prime}\left|\left\langle\psi_{i}(\mathbf{r}) \psi_{i}^{*}\left(\mathbf{r}^{\prime}\right)\right\rangle\right|^{2} V_{\mathrm{TF}}\left(\mathbf{r}-\mathbf{r}^{\prime}\right)
\end{aligned}
$$

We will make the simplifying assumption that the nanoparticle is a billiard so that the average density is constant: $\left\langle|\psi(\mathbf{r})|^{2}\right\rangle=1 / A$ where $A$ is the area of the particle and the correlation function is given by Eq. (13). In this case the mean values are

$$
\begin{aligned}
\left\langle M_{i j}\right\rangle & =\frac{\Delta}{2}+\delta_{i j} \frac{\left\langle\hat{V}_{\mathrm{TF}}\right\rangle_{\mathrm{fs}}}{A} \\
\left\langle N_{i \neq j}\right\rangle & =\frac{\left\langle\hat{V}_{\mathrm{TF}}\right\rangle_{\mathrm{fs}}}{A}
\end{aligned}
$$

where

$$
\left\langle\hat{V}_{\mathrm{TF}}\right\rangle_{\mathrm{fs}}=\int_{0}^{2 \pi} \frac{d \theta}{2 \pi} \hat{V}_{\mathrm{TF}}\left(k_{F} \sqrt{2(1+\cos \theta)}\right)
$$

is the average on the Fermi surface of $\hat{V}_{\mathrm{TF}}\left(\mathbf{k}-\mathbf{k}^{\prime}\right)$. In the zero-range interaction limit, these expressions simplify to

$$
\left\langle M_{i j}\right\rangle=\left(1+\delta_{i j}\right) \Delta / 2, \quad\left\langle N_{i \neq j}\right\rangle=\Delta / 2 .
$$

The magnitude of the residual interaction effects is thus of order the mean level spacing $\Delta$. (Note that the $\Delta$ that we use here and throughout this paper is the spacing of the orbital levels alone and so does not take into account the spin degeneracy factor.) Since energies of this order are critical in determining the spacing of CB peaks - note, for instance, the case of standard up-down filling Eq. (15) - the residual interaction terms must be included in any theory. In particular, the reason for the failure of the simple constant interaction model, Eqs. (11) and (3), is now clear: it does not consistently keep all terms of order $\Delta$.

The variance or covariance of any of the $M_{i j}$ and $N_{i j}$ will involve the correlation function of a wavefunction. For example, consider the variance of $M_{i \neq j}$ :

$$
\begin{aligned}
\operatorname{var}\left(M_{i \neq j}\right)= & \int d \mathbf{r}_{1} d \mathbf{r}_{2} d \mathbf{r}_{3} d \mathbf{r}_{4}\left\langle\left[\left|\psi_{i}\left(\mathbf{r}_{1}\right)\right|^{2}-A^{-1}\right] V_{\mathrm{TF}}\left(\mathbf{r}_{1}-\mathbf{r}_{2}\right)\left[\left|\psi_{j}\left(\mathbf{r}_{2}\right)\right|^{2}-A^{-1}\right]\right. \\
& \left.\times\left[\left|\psi_{i}\left(\mathbf{r}_{3}\right)\right|^{2}-A^{-1}\right] V_{\mathrm{TF}}\left(\mathbf{r}_{3}-\mathbf{r}_{4}\right)\left[\left|\psi_{j}\left(\mathbf{r}_{4}\right)\right|^{2}-A^{-1}\right]\right\rangle \\
= & \int d \mathbf{r}_{1} d \mathbf{r}_{2} d \mathbf{r}_{3} d \mathbf{r}_{4} V_{\mathrm{TF}}\left(\mathbf{r}_{1}-\mathbf{r}_{2}\right) V_{\mathrm{TF}}\left(\mathbf{r}_{3}-\mathbf{r}_{4}\right)\left|\left\langle\psi_{i}\left(\mathbf{r}_{1}\right) \psi_{i}^{*}\left(\mathbf{r}_{3}\right)\right\rangle\right|^{2}\left|\left\langle\psi_{j}\left(\mathbf{r}_{2}\right) \psi_{j}^{*}\left(\mathbf{r}_{4}\right)\right\rangle\right|^{2} .
\end{aligned}
$$

The first equality is due to the normalization of the wavefunction, and the second derives from Eq. (15). Because of the decay of the autocorrelation of $\psi$, Eq. (13), the spatial integrals in (21) are restricted to a region where $\mathbf{r}_{1}$ is close to $\mathbf{r}_{3}$ and likewise $\mathbf{r}_{2}$ to $\mathbf{r}_{4}$. These restrictions produce factors of the small parameter $1 / k_{F} L$ compared to the mean values. We will evaluate these factors below. However, for the large dot limit here, we see that to zeroth order in $1 / k_{F} L$ all the fluctuations in the residual interactions may be neglected 43 45, as in the diffusive case [56 59]. Qualitatively, this is natural because of the averaging implicit in the integral in $M_{i j}$ and $N_{i j}$ : when the size of the system is much larger than the scale of oscillation of $\psi(\mathbf{r})$, particular features of $\psi_{i}$ and $\psi_{j}$ become less important compared to the mean behavior.

For the CB peak spacing distribution, we thus consider the expression for the ground state energy (9) with constant $\left\langle M_{i j}\right\rangle$ and $\left\langle N_{i j}\right\rangle$. The problem is still complicated because of the interplay of the fluctuations in the single particle levels with these constants, which may, for instance, lead to $S=1$ or higher ground state spins 43 45. We thus evaluate the distribution of Coulomb blockade peak spacings numerically: Hamiltonians are taken at random in the GUE ensemble, and the lowest energy states for $N-1, N$, and $N+1$ particles are ob- tained by determining in each case the occupation numbers $\left\{n_{i \sigma}\right\}$ which minimize the expression Eq. (9).

The resulting distribution of $\mathrm{CB}$ peak spacings is shown in Fig. 1. We show it for two different strengths of interactions, the case of $r_{s}=1$ and the zero-range interaction limit. The first thing to note is that for both of these values the distribution differs substantially from that in the CI model, Eq. (3), with much less odd/even alternation present. Still, there is a $\delta$-function in the distribution, as is clear in the cumulative distribution functions in panel (c), because the constant residual interaction term gives a rigid shift to the spacing for odd $N$ and standard filling, Eq. (16). The origin of spacing in these plots is $\Delta^{2} E_{\mathrm{GTF}}+\left\langle M_{i \neq j}\right\rangle$. This is the mean spacing for adding a particle into the next available state neglecting all spin/filling effects; it thus corresponds to the classical charging energy $e^{2} / C$.

The fluctuations of the peak spacings are smaller in this constant exchange model than in the CI model. This is natural since the even and odd parts of the distribution are brought closer together. The values are $\operatorname{rms}\left(\Delta^{2} E_{N}\right) / \Delta=0.58$ in the CI model and $0.28(0.24)$ in the present large dot case for $r_{s}=1$ (zero-range interaction). 

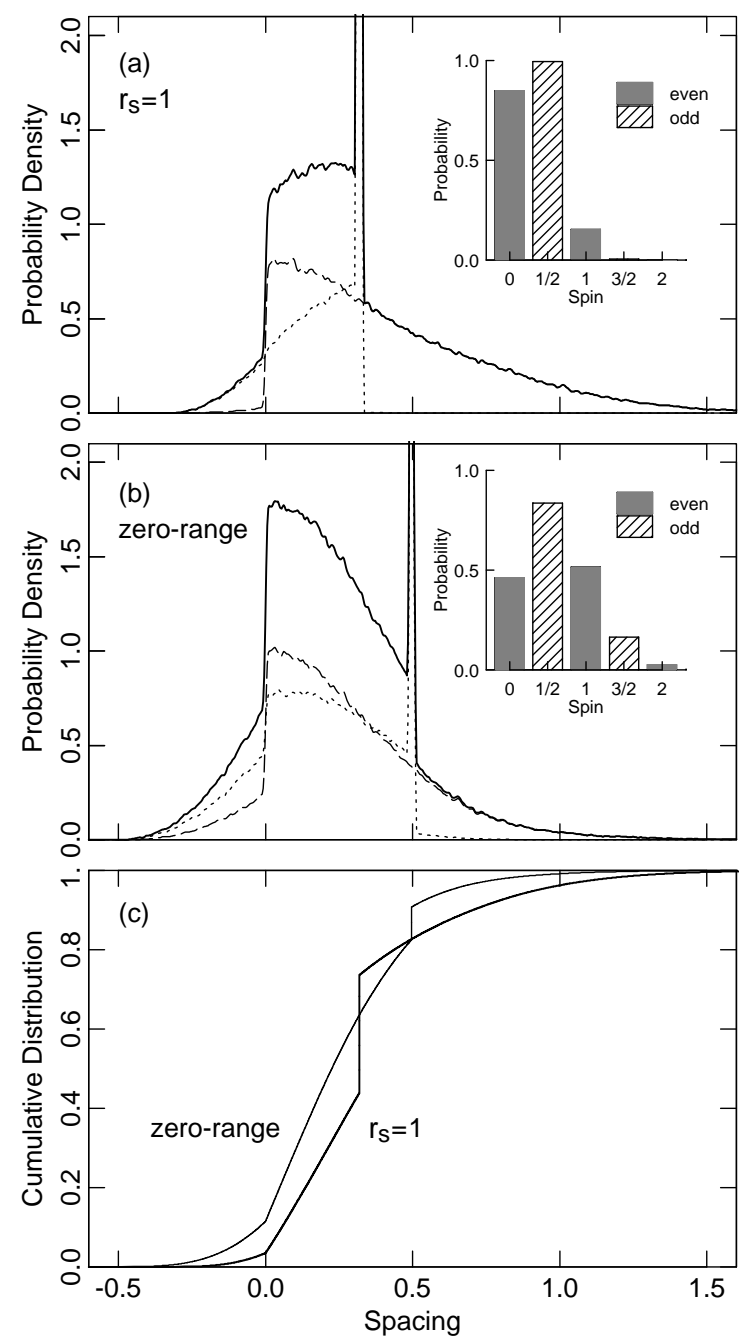

FIG. 1. The probability density of CB peak spacings in the large dot limit $\left(k_{F} L \rightarrow \infty\right)$. The total distribution (solid) as well as that for $N$ even (dashed) and odd (dotted) is given for two strengths of the interactions: (a) $r_{s}=1$ and (b) the zero-range interaction limit. The presence of a $\delta$-function in the distribution is particularly clear in the cumulative distribution functions (the integral of the probability density) shown in (c). Insets show the probability of occurrence of ground state spins in the two cases. The spacing is in units of the mean level separation $\Delta$, and the origin corresponds to the classical spacing $e^{2} / C$.

\section{FLUCTUATIONS OF RESIDUAL INTERACTIONS}

In order to evaluate the statistical effect of fluctuating residual interactions, we need to find the distribution of the $M_{i j}$ and $N_{i j}$. We will show that in the limit of large but not infinite $k_{F} L$, the $M$ 's and $N$ 's are Gaussian distributed and uncorrelated.

The first step is to evaluate the variance of the $M$ 's and $N$ 's. Using the random matrix ensemble introduced in Section [1], we find to a good approximation,

$$
\begin{aligned}
\operatorname{var}\left(M_{i \neq j}\right) & \simeq \frac{8}{\pi A^{2}} \int_{\pi / L}^{2 k-\pi / L} \frac{d q}{q} \frac{V_{\mathrm{TF}}(q)^{2}}{(2 k L)^{2}-(q L)^{2}} \simeq \frac{1}{\pi A^{2}} \frac{\ln (k L)}{(k L)^{2}}\left[2 \hat{V}_{\mathrm{TF}}^{2}(0)+\hat{V}_{\mathrm{TF}}^{2}(2 k)\right] \\
\operatorname{var}\left(N_{i \neq j}\right) & \simeq \frac{2}{\pi A^{2}} \int_{\pi / L}^{2 k-\pi / L} \frac{d q}{q} \frac{\left[\hat{V}_{\mathrm{TF}}(q)+\hat{V}_{\mathrm{TF}}(2 k)\right]^{2}}{(2 k L)^{2}-(q L)^{2}} \\
\left\langle M_{i \neq j} N_{i \neq j}\right\rangle-\left\langle M_{i \neq j}\right\rangle\left\langle N_{i \neq j}\right\rangle & \simeq \frac{4}{\pi A^{2}} \int_{\pi / L}^{2 k-\pi / L} \frac{d q}{q} \frac{\hat{V}_{\mathrm{TF}}(q)\left[\hat{V}_{\mathrm{TF}}(q)+\hat{V}_{\mathrm{TF}}(2 k)\right]}{(2 k L)^{2}-(q L)^{2}}
\end{aligned}
$$


details for the case of $\operatorname{var}\left(M_{i \neq j}\right)$ are given in Appendix A. The diagonal part of the direct residual interaction has an extra contribution because of the additional way in which the wavefunctions can be paired:

$$
\operatorname{var}\left(M_{i i}\right) \simeq 2 \operatorname{var}\left(M_{i \neq j}^{2}\right)+\frac{8}{\pi A^{2}} \int_{\pi}^{2 k-\pi / L} \frac{d q}{q} \frac{\hat{V}_{\mathrm{TF}}(q)\left[\hat{V}_{\mathrm{TF}}(0)+\hat{V}_{\mathrm{TF}}\left(\sqrt{(2 k)^{2}-q^{2}}\right)\right]}{(2 k L)^{2}-(q L)^{2}} .
$$

In the zero-range interaction limit, the expressions for the variance of the $M$ 's and $N$ 's simplify considerably, as for the mean values given in Eq. (20). In this case, we find

$$
\operatorname{var}\left(M_{i j}\right)=\operatorname{var}\left(N_{i \neq j}\right)=\frac{3 \Delta^{2}}{4 \pi} \frac{\ln (k L)}{(k L)^{2}}\left(1+3 \delta_{i j}\right) .
$$

Note that the decay of the wavefunction correlations appearing in the variance produces a factor of $1 / k_{F} L$ in the rms compared to the mean. The $\ln (k L)$ factor is special to two dimensions; it comes from the $1 / k L$ decay of the wavefunction correlator in this case. Thus in the large dot limit, $k_{F} L \gg 1$, the distributions of the $M$ 's and $N$ 's are narrowly peaked about their mean values. This is the justification for ignoring these fluctuations to a first approximation in the last section.

Higher moments of the distribution involve coupling between more pairs of wavefunctions. The non-Gaussian part of the distribution is described by the cumulants, in which all of the wavefunctions are coupled in ways not present in the lower moments. In this case, the spatial integrals will have more restrictions coming from the wavefunction correlation (13); these restrictions will in turn produce additional factors of the small parameter $1 / k_{F} L$. Thus in the large dot limit all higher moments can be neglected. In the case of third and fourth moments this can be easily verified by explicit calculation. Likewise it is straightforward to check that cross correlations among the $M$ 's and $N$ 's do not need to be considered, except for the obvious correlation between $M_{i j}$ and $N_{i j}$ which are equal in the zero-range interaction limit. We shall return to this point in Section VI. In this Section, however, we shall either take $M_{i j}=N_{i j}$ in the zero-range interaction limit, or neglect this correlation for $r_{s}=1$,
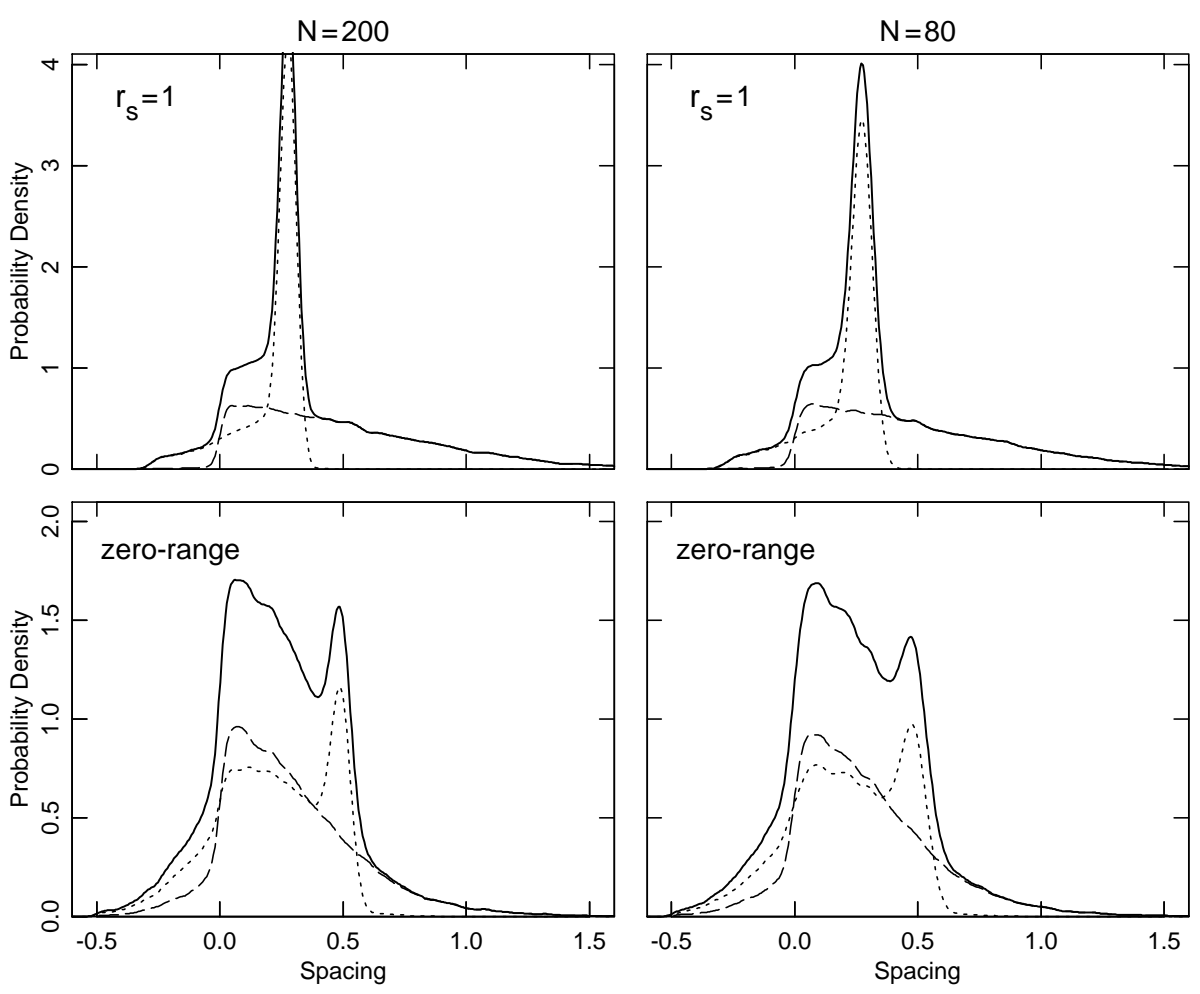

FIG. 2. The probability density of CB peak spacings including the fluctuations in the residual interactions. The total distribution (solid) as well as that for $N$ even (dashed) and odd (dotted) is given for two sizes of quantum dot $(N \approx 200$, left column, or 80 , right column) and two strengths of interaction $\left(r_{s}=1\right.$, top row, or the case of a zero-range interaction, bottom row). All of the features of the distribution are smoothed compared to Fig. 1 . Note that the odd/even effect is quite clear in all four cases, and that the remnants of the $\delta$-function are discernible. The spacing is in units of the mean level separation $\Delta$, and the origin corresponds to the classical spacing $e^{2} / C$. 
and therefore take the $M_{i j}$ and $N_{i j}$ to be uncorrelated random Gaussian variables with mean and variance given above.

We now use the statistics of the $M_{i j}, N_{i j}$, and single particle levels (assumed to follow the random matrix GUE ensemble as in Section III) to deduce the statistics of the CB peak spacings. It is clear from the expressions for the spacing in the simple up-down filling case, Eq. (15), that sums such as $\sum_{j} M_{i j}$ will be important. Because of the saturation of the fluctuations, item iii) in the random matrix model of Section II, the variance of such a sum involves only levels within $E_{\mathrm{Th}}$ of each other. In calculating $\operatorname{var}\left(\sum_{j} M_{i j}\right)$, then, the sum should be taken over of order $k_{F} L$ independent $M$ 's:

$$
\operatorname{var}\left(\sum_{j} M_{i j}\right) \propto \frac{\ln \left(k_{F} L\right)}{k_{F} L} \Delta^{2} .
$$

The variance of other sums over the residual interaction terms will have the same dependence on $k_{F} L$. Up to a $\sqrt{\ln }$ factor, we thus expect the contribution of the fluctuations of the residual interactions to the $C B$ peak spacings to be of order $\Delta / \sqrt{k_{F} L}$ in rms.

In order to evaluate the magnitude more accurately, we turn again to numerical evaluation. We consider a collection of $E_{\mathrm{Th}} / \Delta$ levels with GUE energies and Gaussian residual interaction terms. We fill the levels with $N-1$, $N$, and $N+1$ particles successively, and find the occupation numbers $\left\{n_{i \sigma}\right\}$ minimizing the energy Eq. (9) in each case. Fig. 2 shows the resulting distribution of CB peak spacings. The fluctuations clearly act to smooth out all the sharp features of the $k_{F} L \rightarrow \infty$ case: the $\delta$-function becomes a finite peak, and the discontinuity at the origin is removed. The difference between the $N$ even and odd cases is quite clear - even for the relatively small $N=80$ (for which $1 / \sqrt{k_{F} L} \simeq 0.21$ ). The fluctuations of the residual interaction terms lead, of course, to an increase in the fluctuations of the spacing; now $\operatorname{rms}\left(\Delta^{2} E_{N}\right) / \Delta=0.31$ for $r_{s}=1$ and $N=80-200$ compared with 0.28 in the absence of such fluctuations. While the inclusion of the fluctuations has clearly made the distributions more like the experiments, substantial differences remain.

\section{SCRAMBLING: ADDED ELECTRON CHANGES CONFINEMENT}

As electrons are added to the nanoparticle, the mean field potential which confines the electrons changes. This causes a change in the single particle levels, whose energies appear in the expression for the ground state energy Eq. (9), which then in turn change the CB peak spacing. Because we consider a chaotic quantum dot, the change in the energy levels will be unpredictable; hence, we call this effect "scrambling". Note that it is an intrinsic effect connected to the charge of the electron not the geometry or environment of the dot. This effect has been studied previously for diffusive quantum dots in Ref. 42]; here we give a derivation directly in the context of ballistic chaotic dots.

We know from the Strutinsky approach (Section II) that the change in the single particle levels should be found from the change in the smooth Thomas-Fermi potential, Eq. (7). So we first evaluate this change in potential, and then use perturbation theory to find the change in the energy levels. Perturbation theory is justified since the resulting shift is smaller than the mean level separation $\Delta$ by a factor depending on $k_{F} L$. As a check, one can evaluate the magnitude of the fluctuation in the event of a complete scrambling of all the single particle levels. The properties of sums of random matrix eigenvalues in Ref. [60 imply a very large fluctuation of the peak spacings - of the order $2-4 \Delta$ which is larger than observed experimentally.

\section{A. Evaluation of $\delta \mathbf{V}_{\text {eff }}$}

The generalized Thomas-Fermi problem is specified in terms of the density functional

$$
\mathcal{F}_{\mathrm{GTF}}[n]=\mathcal{T}_{\mathrm{TF}}[n]+\mathcal{E}_{\text {ext }}[n]+\mathcal{E}_{\text {coul }}[n]+\mathcal{E}_{\mathrm{xc}}[n]
$$

comprising four contributions: the kinetic energy

$$
\mathcal{T}_{\mathrm{TF}}[n]=\int d \mathbf{r}\left[\int^{n(\mathbf{r})} d n^{\prime} \epsilon\left(n^{\prime}\right)\right]
$$

where $\epsilon\left(n^{\prime}\right)$ is the maximum kinetic energy of noninteracting particles with density $n^{\prime}$, the confinement potential defining the geometry of the dot

$$
\mathcal{E}_{\text {ext }}[n]=\int d \mathbf{r} V_{\text {ext }}(\mathbf{r}) n(\mathbf{r}),
$$

the Coulomb energy

$$
\mathcal{E}_{\text {coul }}[n]=\frac{e^{2}}{2} \int d \mathbf{r} d \mathbf{r}^{\prime} \frac{n(\mathbf{r}) n\left(\mathbf{r}^{\prime}\right)}{\left|\mathbf{r}-\mathbf{r}^{\prime}\right|},
$$

and the exchange-correlation potential $\mathcal{E}_{\mathrm{xc}}$. The ThomasFermi density is found by solving

$$
\frac{\delta \mathcal{F}_{\mathrm{GTF}}}{\delta n}\left[n_{\mathrm{GTF}}\right]=\mu_{T F},
$$

where $n(\mathbf{r})$ is the electronic density and $\mu_{T F}$ is found such that $\int n(\mathbf{r}) d \mathbf{r}=N$. The effective potential is, then, defined by

$$
V_{\mathrm{eff}}(\mathbf{r}) \equiv \frac{\delta\left(\mathcal{E}_{\mathrm{ext}}+\mathcal{E}_{\mathrm{coul}}+\mathcal{E}_{\mathrm{xc}}\right)}{\delta n}\left[n_{\mathrm{GTF}}\right] .
$$

Let $\delta n_{\mathrm{GTF}}=n_{\mathrm{GTF}}(N+1)-n_{\mathrm{GTF}}(N)$ be the change in the Thomas-Fermi density when one electron is added to 
the system. Bearing in mind that Eq. (33) is a classicallike equation for which $\delta n_{\mathrm{GTF}}$ is small, one can write

$$
\begin{aligned}
\int d \mathbf{r}^{\prime} \frac{\delta^{2} \mathcal{T}_{\mathrm{TF}}}{\delta n^{2}}[ & \left.n_{\mathrm{GTF}}\right]\left(\mathbf{r}, \mathbf{r}^{\prime}\right) \delta n_{\mathrm{GTF}}\left(\mathbf{r}^{\prime}\right) \\
& +\int d \mathbf{r}^{\prime} \frac{\delta V_{\mathrm{eff}}}{\delta n}\left(\mathbf{r}, \mathbf{r}^{\prime}\right) \delta n_{\mathrm{GTF}}\left(\mathbf{r}^{\prime}\right)=\delta \mu_{T F}
\end{aligned}
$$

with $\delta \mu_{T F}$ such that $\int \delta n_{\mathrm{GTF}}(\mathbf{r})=1$. In two dimensions, the Coulomb energy of the added charge will dominate any variations in the kinetic energy. One can therefore write the variation of the density as $\delta n_{\mathrm{GTF}}=$ $\delta n_{\mathrm{GTF}}^{0}+\delta n_{\mathrm{GTF}}^{1}+\ldots$ with $\delta n_{\mathrm{GTF}}^{0}$ the solution of the electrostatic problem

$$
\begin{aligned}
\delta \mu^{0} & =\int d \mathbf{r}^{\prime} \frac{\delta^{2} \mathcal{E}_{\mathrm{coul}}}{\delta n^{2}}\left(\mathbf{r}, \mathbf{r}^{\prime}\right) \delta n_{\mathrm{GTF}}^{0}\left(\mathbf{r}^{\prime}\right) \\
& =e^{2} \int d \mathbf{r}^{\prime} \frac{\delta n_{\mathrm{GTF}}^{0}\left(\mathbf{r}^{\prime}\right)}{\left|\mathbf{r}-\mathbf{r}^{\prime}\right|}
\end{aligned}
$$

At this level of approximation, the variation of the potential inside the dot is just the constant $\delta \mu^{0}$, which will just shift the one particle energies $\epsilon_{i}$ but not give rise to any fluctuation. One should keep in mind though that Eq. (33) applies only at places where $n_{\mathrm{GTF}}(\mathbf{r}) \neq 0$. Therefore the boundaries of the effective potential can be affected, in a way that depends, of course, largely on the external confining potential, and in particular on the second derivative of this latter at the the boundary. If the curvature is weak, fairly large displacements of the boundary can occur, which could play a significant role.

If, on the other hand, one assumes a billiard-like confining potential, the only source of modification of the effective potential comes from $\delta n_{\mathrm{GTF}}^{1}$, which is obtained through the equation

$$
\int d \mathbf{r}^{\prime} \frac{\delta^{2} \mathcal{T}_{\mathrm{TF}}}{\delta n^{2}}\left(\mathbf{r}, \mathbf{r}^{\prime}\right) \delta n_{\mathrm{GTF}}^{0}\left(\mathbf{r}^{\prime}\right)+\int d \mathbf{r}^{\prime} \frac{\delta V_{\mathrm{eff}}}{\delta n}\left(\mathbf{r}, \mathbf{r}^{\prime}\right) \delta n_{\mathrm{GTF}}^{1}=\delta \mu^{1} .
$$

Noting that $\int\left(\delta V_{\text {eff }} / \delta n\right) \delta n_{\text {GTF }}^{1}$ is, up to an inessential additive constant, the variation $\delta V_{\text {eff }}$ of the effective potential we are interested in, and that

$$
\frac{\delta^{2} \mathcal{T}_{\mathrm{TF}}}{\delta n^{2}}\left(\mathbf{r}, \mathbf{r}^{\prime}\right)=\nu^{-1} \delta_{d}\left(\mathbf{r}-\mathbf{r}^{\prime}\right)
$$

we get

$$
\delta V_{\mathrm{eff}}(\mathbf{r})=-\Delta(\delta \tilde{n}(\mathbf{r})-\overline{\delta \tilde{n}})
$$

where $\delta \tilde{n} \equiv A \delta n_{\mathrm{GTF}}^{0}(\mathbf{r})$ is a smooth function of order 1 ( $A$ is the area of the dot). Note that we choose $\delta V_{\text {eff }}$ with mean value zero. This ensures that there is no change in the mean energy levels; any such change in the mean should be incorporated in the charging energy.

\section{First order terms}

\section{B. Consequences for the peak spacing distribution}

The variation of the effective potential induces a change of the one particle energies. We let $\varepsilon_{N}$ denote the single particle energy of the $N^{\text {th }}$ electron, whatever orbital it is in (for $N$ even and simple up/down filling, for instance, $\left.\varepsilon_{N}=\epsilon_{N / 2}\right)$. In perturbation theory, $\delta \varepsilon_{j}=\delta \varepsilon_{j}^{(1)}+\delta \varepsilon_{j}^{(2)}+\ldots$ with

$$
\begin{aligned}
\delta \varepsilon_{j}^{(1)} & =\left\langle\psi_{j}\left|\delta V_{\mathrm{eff}}\right| \psi_{j}\right\rangle \\
\delta \varepsilon_{j}^{(2)} & =\sum_{k \neq j} \frac{\left|\left\langle\psi_{j}\left|\delta V_{\mathrm{eff}}\right| \psi_{k} \mid\right\rangle\right|^{2}}{\varepsilon_{k}-\varepsilon_{j}} .
\end{aligned}
$$

When going back to the inverse compressibility, one should bear in mind, however, that the variation of $V_{\text {eff }}$ when going from $N-1$ to $N$ electron is the same as when going from $N$ to $N+1$. As a consequence, it can be checked easily that the linear variation $\delta \varepsilon_{j}^{(1)}$ cancels for all levels except the two top ones. At this level of approximation one therefore gets

$$
\Delta^{2} E_{N}=\Delta^{2} E_{N}^{\mathrm{CI}}+\Delta^{2} E_{N}^{\text {scramb }}
$$

where $\Delta^{2} E_{N}^{\mathrm{CI}}$ is the constant interaction model result given in Eq. (3), and

$$
\Delta^{2} E_{N}^{\text {scramb }}=\delta \varepsilon_{N+1}+\delta \varepsilon_{N}+\sum_{j=1}^{N-1} \delta^{2} \varepsilon_{j}
$$

is the correction due to scrambling. The levels used for $\Delta^{2} E_{N}^{\mathrm{CI}}$ are those for the $N$-electron $V_{\text {eff }}$. Throughout this scrambling argument we assume that the filling of the levels does not change.

The mean of the first order terms is zero by construction. The variance of $\delta \varepsilon_{i}^{(1)}$ is

$$
\operatorname{var}\left(\delta \varepsilon_{i}^{(1)}\right)=\Delta^{2} \int d \mathbf{r} d \mathbf{r}^{\prime}\left|\left\langle\psi_{i}(\mathbf{r}) \psi_{i}^{*}\left(\mathbf{r}^{\prime}\right)\right\rangle\right|^{2} \delta \tilde{n}(\mathbf{r}) \delta \tilde{n}\left(\mathbf{r}^{\prime}\right) .
$$

Using the wavefunction correlations (13) and approximating $J_{0}^{2}\left(k_{F}\left|\mathbf{r}-\mathbf{r}^{\prime}\right|\right) \simeq 1 /\left(\pi k_{F}\left|\mathbf{r}-\mathbf{r}^{\prime}\right|\right)$ plus oscillating terms $\left(k_{F}\left|\mathbf{r}-\mathbf{r}^{\prime}\right|>1\right)$, we find

$$
\operatorname{var}\left(\delta \varepsilon_{i}\right) \simeq \alpha \cdot \frac{\Delta^{2}}{\pi k_{F} L} .
$$

The prefactor depends on the geometry of the dot under consideration:

$$
\alpha=\int d^{2} \mathbf{u} d^{2} \mathbf{u}^{\prime} \frac{1}{\left|\mathbf{u}-\mathbf{u}^{\prime}\right|} \delta \tilde{n}(\mathbf{u}) \delta \tilde{n}\left(\mathbf{u}^{\prime}\right)
$$


is a dimensionless quantity of order 1 (note $\mathbf{u}=\mathbf{r} / L$ ). For a circular dot, for instance, with diffuse boundary scattering to make it chaotic, $\delta \tilde{n}=\left\{\left[1-(r / R)^{2}\right]^{-1 / 2}-2\right\} / 2$ where $R$ is the radius; in this case, $\alpha \simeq 1.2$.

Bearing in mind that $k_{F} L \simeq \sqrt{2 \pi N}$ (taking into account the spin degeneracy), we see that the typical amplitude of the variation of the $\delta \varepsilon_{i}$ scales as $N^{-1 / 4}$ times the mean level spacing. This may, in fact, be numerically significant for experimental dots - for a circular dot with 100 electrons, for instance, the magnitude would be $\simeq 0.12 \Delta$.

\section{Second order terms}

The variance of the sum $\sum_{1}^{N-1} \delta^{2} \varepsilon_{i}$ can be evaluated along the same lines. Since it turns out to be parametrically smaller, we will be less careful with the constant.

First we note that the fluctuation of an individual matrix element is

$\operatorname{var}\left(\left\langle i\left|\delta V_{\text {eff }}\right| j \mid\right\rangle\right)=\frac{\Delta^{2}}{A^{2}} \int d \mathbf{r} d \mathbf{r}^{\prime} J_{0}\left(k_{i} \delta \mathbf{r}\right) J_{0}\left(k_{j} \delta \mathbf{r}\right) \delta \tilde{n}(\mathbf{r}) \delta \tilde{n}\left(\mathbf{r}^{\prime}\right)$ where $\delta \mathbf{r}=\mathbf{r}-\mathbf{r}^{\prime}$. Following the same reasoning as above, this implies that

$$
\operatorname{var}\left(\left\langle i\left|\delta V_{\text {eff }}\right| j \mid\right\rangle\right) \simeq\left\{\begin{array}{ccc}
0 & \text { if } & \delta k L>1 \\
\alpha \Delta^{2} / \pi k_{F} L & \text { if } & \delta k L<1
\end{array}\right.
$$

where $\delta k=k_{i}-k_{j}$.

This result is now inserted in the expression (41) for the energy shift $\delta \varepsilon_{j}^{(2)}$ and then for the inverse compressibility Eq. (43). Assuming the matrix elements $\left\langle i\left|\delta V_{\text {eff }}\right| j \mid\right\rangle$ are Gaussian distributed independent variables, we find

$$
\begin{aligned}
\sigma_{2}^{2} & \equiv \operatorname{var}\left(\sum_{i=1}^{N-1} \delta^{2} \varepsilon_{i}\right) \\
& \simeq \sum_{i=N-N_{c}}^{N-1} \sum_{j=N}^{N+N_{c}} \frac{1}{\left(\bar{\varepsilon}_{i}-\bar{\varepsilon}_{j}\right)^{2}} \operatorname{var}\left(\left|\left\langle\psi_{i}\left|\delta V_{\mathrm{eff}}\right| \psi_{j} \mid\right\rangle\right|^{2}\right) .
\end{aligned}
$$

Because of the saturation of the fluctuations of the levels, point (iii) in the random matrix criteria of Section II, the sum over levels is cut off at $N_{c}=k_{F} L$. The final result is

$$
\sigma_{2}^{2} \propto \ln k_{F} L\left(\frac{\Delta}{k_{F} L}\right)^{2}
$$
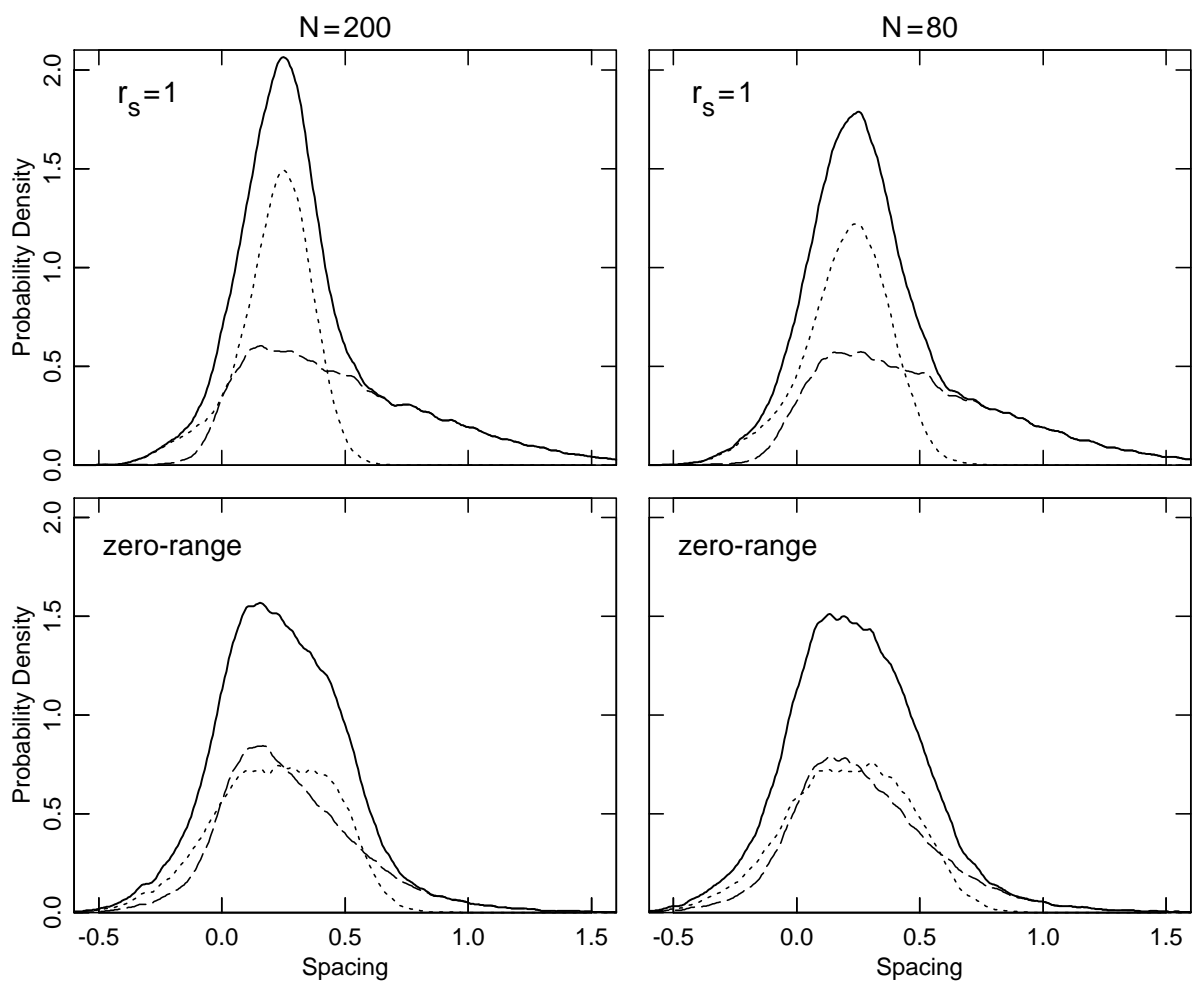

FIG. 3. The probability density of CB peak spacings including the scrambling effect on the top two levels as well as the fluctuations in the residual interactions. The total distribution (solid) as well as that for $N$ even (dashed) and odd (dotted) is given for two sizes of quantum $\operatorname{dot}\left(N=200\right.$, left column, or 80 , right column) and two strengths of interaction $\left(r_{s}=1\right.$, top row, or the case of a zero-range interaction, bottom row). Note that the clear odd/even effect for $r_{s}=1$ and the relatively small $N=80$ remains visible even for the stronger zero-range interaction. The spacing is in units of the mean level separation $\Delta$, and the origin corresponds to the classical spacing $e^{2} / C$. 
which is parametrically smaller than the first order result (45). We therefore see that the the effect of scrambling is dominated by the variation of the top levels, and that this adds contributions to the peak spacing fluctuations which while parametrically small on the scale of $\Delta$ could be numerically important for dots which are not too large.

\section{Numerics}

It is a simple matter to incorporate the scrambling of the top two levels into our numerical investigation of the ground state energy specified by Eq. (9). In random matrix theory, the change in an energy level upon varying a parameter is uncorrelated with its value 61, 62, so we simply choose random $\delta \varepsilon_{N}$ and $\delta \varepsilon_{N+1}$ from a Gaussian distribution with variance Eq. (45). The ground state configuration is then found as before.

Fig. 3 shows the resulting probability density of $\mathrm{CB}$ peak spacings. In the $r_{s}=1$ case there is still a substantial difference between the distributions of even and odd spacings: for $N$ even, the large spacing tail coming from the level-spacing distribution (Wigner surmise) survives even for less than 100 electrons. When the interactions are stronger, as in the zero-range interaction case shown, the even and odd distributions are more similar, but there is still a discernible difference. The magnitude of the fluctuations increases further: we now find $\operatorname{rms}\left(\Delta^{2} E_{N}\right) / \Delta=0.33-0.32$ for $r_{s}=1$ and $N=80-200$.

\section{BEYOND THE GAUSSIAN MODEL}

Our treatment of the fluctuations of the residual interactions in the previous sections relies on a certain number of assumptions. We have for instance neglected correlations among the $M_{i j}$ and $N_{i^{\prime} j^{\prime}}$ (except for the obvious $M_{i j}=N_{i j}$ for a zero-range interaction) when we know, for instance, that the correlation coefficient of $M_{i j}$ with $N_{i j}$ is of order 1 even in the infinite size limit. Similarly we have assumed the absence of correlation between these residual interaction terms and the one particle energies $\epsilon_{i}$. Furthermore, we have used expressions such as Eqs. (23)(26) which are derived in the large $k_{F} L$ limit. To check that these assumptions do not drastically modify the resulting distribution of CB peak spacings, we shall in this section implement the random matrix ensemble described in Section II [paragraph containing Eq. (12)] by directly performing numerical Monte Carlo calculations.

To implement the constraints of our random matrix model, we start by considering the system to be a square of size $L$, for which we can use a plane wave basis

$$
\left|n_{x}, n_{y}\right\rangle=L^{-2} \exp \left[i 2 \pi n_{x} x / L\right] \exp \left[i 2 \pi n_{y} y / L\right]
$$

ordered by increasing momentum. In this basis, we write the one particle Hamiltonian as $H=D+V$ where $D$ is diagonal with $D_{i i}=i$. $V$ is taken to be a banded random Hamiltonian with independent complex matrix elements which are Gaussian distributed with variance $v_{i j}^{2}=a(i) \exp (-b(i)|i-j|)$. For $a(i)$ in the correct range 63. such an ensemble yields (1) eigenvalues with a mean spacing close to one, (2) local fluctuations which are typical of the GUE, and (3) eigenfunctions which are localized in energy. The localization in energy can be characterized by the inverse participation ratio in the plane wave basis: $I_{j}=\sum_{n_{x}, n_{y}}\left|\left\langle n_{x}, n_{y} \mid \epsilon_{j}\right\rangle\right|^{4}$. Our requirement Eq. (12) implies the constraint

$$
I_{j}^{-1}=(3 / 4)\left(k_{j} L\right) \text {. }
$$

In practice, we use a simple algorithm to tune the value of $a(i)$ and $b(i)$ in such a way that this constraint holds. Diagonalizing the random matrix specified leads to eigenvalues and eigenvectors which are then used to evaluate ground state energies for different number of particles via Eq. (9), and hence arrive at the CB peak spacings in this model.

In this approach, deviations from the wavefunction correlations (13) required by normalization are naturally present. Furthermore, correlation between eigenvalues and eigenfunctions is present as well. Of course, in a real physical system, these effects would be automatically included because of the scattering at the boundary of the system - the transit time $\tau$, for instance, gives both the scale over which fluctuations saturate $(\hbar / \tau)$ and the length scale at which the Bessel function correlations become suspect $\left(v_{F} \tau\right)$. We believe that our random matrix model includes the main features of these effects in a generic way.

Fig. 4 shows numerical results for this model. We consider only the smaller value of $N, N=80$, because the effects are expected to be only significant for smaller quantum dots. On the other hand, we present results both with and without the scrambling effect of Section У, and for different interaction strengths $-r_{s}=1$ corresponds to a typical value for a GaAs quantum dot while the zero-range interaction case corresponds to the maximum effect possible within a perturbative approach to the interactions. When these results are compared to those of Figs. 2 and 3, we see that there is a small but noticeable effect. This is highlighted in the quantilequantile plots in the insets to Fig. 4 where an ordered set of RMT spacings is plotted against a similar set for the Gaussian model. The present RMT yields a more pronounced large spacing tail than the Gaussian model.

\section{CONCLUSIONS}

In this paper we have studied the Coulomb blockade peak spacings for large but not infinite quantum dots 

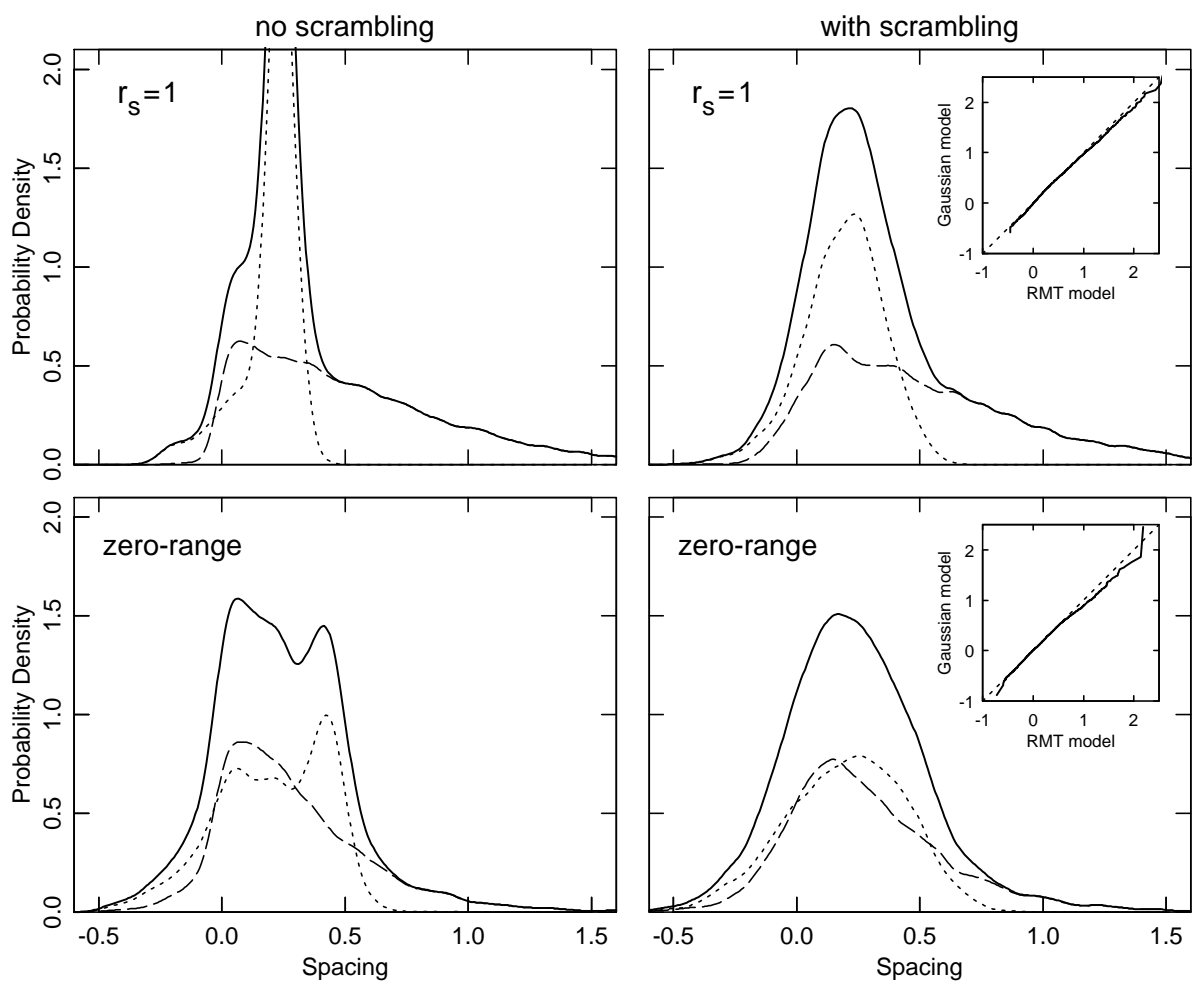

FIG. 4. The probability density of CB peak spacings within a random matrix model which goes beyond the Gaussian approximation. For $N \approx 80$, the total distribution (solid) as well as that for $N$ even (dashed) and odd (dotted) is given for two strengths of interaction $\left(r_{s}=1\right.$, top row, or the case of a zero-range interaction, bottom row) in the presence of scrambling (right column) and without scrambling (left column). Features of the distribution are more pronounced than in the Gaussian model (Figs. 2 and 3), suggesting that smoothing effects are slightly overestimated in the Gaussian approximation. Insets: Quantile-quantile plots of the random matrix model versus the Gaussian model; note the differences at large spacings. Throughout, the spacing is in units of the mean level separation $\Delta$, and the origin corresponds to the classical spacing $e^{2} / C$.

in the case that the interactions can be treated perturbatively and the single-particle properties described by random matrix theory. Motivated by the Strutinsky approach to finite Fermi systems $[50$ 52], we use an expression for the ground state energy [Eq. (9)] which includes the charging energy, the single particle quantization effects, and the residual (screened) interactions treated in first order (Hartree-Fock).

We presented results in four cases. First, in the limit $k_{F} L \rightarrow \infty$, fluctuations of the residual interactions are negligible. The $\mathrm{CB}$ peak spacing distribution has a discontinuity at $e^{2} / C$ and a $\delta$-function because of the odd $N$ case (Fig. 1). The distribution is very different from that predicted by the simple CI model which neglects some terms of order $\Delta$ while keeping others.

Second, fluctuations in the residual interactions contribute a term typically of order $\Delta / \sqrt{k_{F} L}$ to the CB peak spacings. The sharp features of the infinite dot limit are rounded out (Fig. 2).

Third, the addition of an electron in the CB process changes the confinement potential in the quantum dot and so changes the single particle properties. This scrambling effect also contributes a term typically of order
$\Delta / \sqrt{k_{F} L}$ to the CB peak spacings, and the distribution is rounded further. However, the difference between $N$ even and $N$ odd remains substantial at large spacings where a tail is present for $N$ even (Fig. 3) which is a remnant of the Wigner surmise distribution for level spacings.

Finally, we took a first step towards investigating corrections to the Gaussian uncorrelated model for the fluctuations of the residual interactions. We found that the large spacing part of the distribution is somewhat underestimated in the Gaussian model.

Before ending this paper, we compare our theoretical distributions to those obtained experimentally [25 29]. The observed distribution has little structure, being Gaussian near its peak but with longer tails for both larger and smaller spacings. The width of the distribution is contentious: early work claimed that the width scaled with the charging energy 25,26] but this has not been verified by more recent experiments 27 29]. In the more recent experiments, the width is clearly related to the mean level separation $\Delta$ and in the range 0.5-1.5 $\Delta$, somewhat larger than given by, e.g., the CI model.

The theoretical results presented here certainly look more like the experiments than the benchmark CI model: 
the distribution is more rounded and so less structured, and the odd/even alternation is much less pronounced. We suspect that further development of this approach will produce qualitative agreement with the experiments.

In the end, however, the theory presented here does not provide an adequate quantitative description of the experiments. To start with, the magnitude of the fluctuations is actually less than in the CI model ( 0.58 versus 0.32 for $N=200$ ) and so differs from experiment. With regard to the odd/even effect, the situation is somewhat less clear, and so more interesting. Looking at Fig. 3, for instance, one observes that a substantial odd/even effect is still present for $r_{s}=1$, while it has been largely washed out in the zero-range limit of the screened interaction. It should be stressed that this drastic modification of the distributions can be traced back mainly to the relatively modest change in the mean values $\left\langle M_{i i}\right\rangle-\Delta / 2$ and $\left\langle N_{i j}\right\rangle$, Eqs. (18)-(20), which change from about $0.3 \Delta$ for $r_{s}=1$ to $0.5 \Delta$ in the zero-range limit. The sensitivity to the numerical value of these quantities should not come as a surprise since we know that the Stoner instability, for which a spontaneous polarization of the spin of many electrons takes place, occurs for $\left\langle N_{i j}\right\rangle=\Delta$. These observations suggest an experiment: placing a good conductor close to the 2DEG in such a way that it can screen the interaction between electrons within the quantum dot should significantly affect the peak spacing distribution. If the auxiliary screening is strong enough, a strong odd/even effect should be visible. A double quantum well structure seems like a possible setting for such an experiment.

We can divide the potential explanations for the quantitative discrepancy between theory and experiment into basically three categories. The first one, which we cannot absolutely exclude, is that noise in the experiments is corrupting the results [27,64]. The second is that effects not included here, but interpretable within the kind of Fermi liquid theory on which we have relied throughout this paper, dominate. Some candidates are (a) the effect of the changing gate potential on the single particle levels 114,15 - this would, however, somewhat contradict the remarkable stability observed in the wavefunction 65-(b) boundary effects in the scrambling which need to be considered using a realistic description of the dot, and (c) consequences of non-chaotic dynamics within the dots 11, 15. Finally, a third completely different kind of interpretation is that the perturbative approach to interactions taken here is not accurate even though $r_{s} \approx 1-2$ [25,31,40,41. Further experimental and theoretical work is needed to discern between these options.

\section{ACKNOWLEDGEMENTS}

We thank G. Usaj for many valuable conversations, and appreciate helpful discussions with I. L. Aleiner, L. I.
Glazman, and C. M. Marcus. The LPTMS is a "Unité de recherche de l'Université Paris 11 associée au C.N.R.S."

\section{APPENDIX A: VARIANCE OF THE $M_{I J}$}

In this appendix we sketch the argument which leads to Eqs. (23)-(26). As in Section VI, we assume that the configuration space is a square of size $L$, for which the plane wave basis $|\mathbf{k}\rangle=\left|n_{x}, n_{y}\right\rangle$ introduced in Eq. (51) can be used.

Expanding the eigenfunctions in this basis,

$$
\left|\varphi_{i}\right\rangle=\sum_{\mathbf{k}} v_{i \mathbf{k}}|\mathbf{k}\rangle
$$

we can write the $M_{i j}$ 's as

$$
\begin{aligned}
M_{i j} & =\sum_{\mathbf{k}_{1}, \mathbf{k}_{2}, \mathbf{k}_{3}, \mathbf{k}_{4}} v_{i \mathbf{k}_{1}} v_{i \mathbf{k}_{2}}^{*} v_{j \mathbf{k}_{3}} v_{j \mathbf{k}_{4}}^{*} \delta_{\mathbf{k}_{1}-\mathbf{k}_{2},-\mathbf{k}_{3}+\mathbf{k}_{4}} \hat{V}_{\mathrm{TF}}\left(\mathbf{k}_{1}-\mathbf{k}_{2}\right) \\
& =\sum_{\mathbf{q}} \hat{V}_{\mathrm{TF}}(\mathbf{q}) W_{i \mathbf{q}} W_{j \mathbf{q}}^{*}
\end{aligned}
$$

where we define

$$
W_{i \mathbf{q}} \equiv \sum_{\mathbf{k}_{1}-\mathbf{k}_{2}=\mathbf{q}} v_{i \mathbf{k}_{1}} v_{i \mathbf{k}_{2}}^{*} .
$$

Our random matrix model (Section [II) implies

$$
\begin{aligned}
\left\langle v_{i \mathbf{k}_{1}} v_{j \mathbf{k}_{2}}^{*}\right\rangle & =\left(k_{F} L\right)^{-1} \delta_{i j} \delta_{\mathbf{k}_{1} \mathbf{k}_{2}} & & \text { if } \delta k<\pi / L \\
& =0 & & \text { if } \delta k>\pi / L
\end{aligned}
$$

with $\delta k=|| \mathbf{k}_{1}\left|-k_{i}\right|$ and $k_{i}=\sqrt{2 m E_{i}} / \hbar$. From this, we deduce

$$
\operatorname{var}\left(M_{i \neq j}\right) \simeq \frac{1}{A^{2}} \sum_{\mathbf{q} \neq 0} \hat{V}_{\mathrm{TF}}^{2}(\mathbf{q})\left\langle\left|W_{i \mathbf{q}}\right|^{2}\right\rangle\left\langle\left|W_{j \mathbf{q}}\right|^{2}\right\rangle
$$

$\left|W_{j \mathbf{q}}\right|^{2}$ can be interpreted as $\left(2 \pi k_{i}\right)^{-2}$ times the area of the intersection of two rings of diameter $k_{i}$ and width $2 \pi / L$, centered at a distance $|\mathbf{q}|=q$. Simple geometry therefore gives, for $2 \pi / L \leq|\mathbf{q}| \leq 2 k_{i}-2 \pi / L$

$$
\left\langle\left|W_{i \mathbf{q}}\right|^{2}\right\rangle \simeq \frac{4}{(q L) \sqrt{\left(2 k_{i} L\right)^{2}-(q L)^{2}}} .
$$

We obtain for $i \simeq j$

$$
\operatorname{var}\left(M_{i \neq j}\right) \simeq \frac{8}{\pi A^{2}} \int_{\pi / L}^{2 k-\pi / L} \frac{d q}{q} \frac{V_{\mathrm{TF}}(q)^{2}}{(2 k L)^{2}-(q L)^{2}}
$$

The variance of $M_{i i}$ and $N_{i j}$ and the covariance between $M_{i j}$ and $N_{i j}$ can be computed along the same lines.

[1] Single Charge Tunneling: Coulomb Blockade Phenomena in Nanostructures, edited by H. Grabert and M. Devoret (Plenum, New York, 1992). 
[2] Mesoscopic Electron Transport, edited by L. L. Sohn, L. P. Kouwenhoven, and G. Schön (Kluwer, New York, 1997).

[3] D. K. Ferry and S. M. Goodnick, Transport in Nanostructures (Cambridge University Press, New York, 1997).

[4] Nanotechnology, edited by G. Timp (Springer-Verlag/AIP, New York, 1999).

[5] M. A. Kastner, Rev. Mod. Phys. 64, 849 (1992).

[6] L. P. Kouwenhoven, C. M. Marcus, P. L. McEuen, S. Tarucha, R. M. Westervelt, and N. S. Wingreen, in Mesoscopic Electron Transport, edited by L. L. Sohn, L. P. Kouwenhoven, and G. Schön (Kluwer, New York, 1997), pp. 105-214.

[7] D. C. Ralph, C. T. Black, J. M. Hergenrother, J. G. $\mathrm{Lu}$, and M. Tinkham, in Mesoscopic Electron Transport, edited by L. L. Sohn, L. P. Kouwenhoven, and G. Schön (Kluwer, New York, 1997), pp. 447-467.

[8] I. L. Aleiner, P. W. Brouwer, and L. I. Glazman, in preparation (unpublished).

[9] R. A. Jalabert, A. D. Stone, and Y. Alhassid, Phys. Rev. Lett. 68, 3468 (1992).

[10] M. Stopa, Phys. Rev. B 54, 13767 (1996).

[11] M. Stopa, Physica B 251, 228 (1998).

[12] G. Hackenbroich, W. D. Heiss, and H. A. Weidenmüller, Phys. Rev. Lett. 79, 127 (1997).

[13] Y. Alhassid, M. Gökçedag, and A. D. Stone, Phys. Rev. B 58, 7524 (1998).

[14] R. O. Vallejos, C. H. Lewenkopf, and E. R. Mucciolo, Phys. Rev. Lett. 81, 677 (1998).

[15] R. O. Vallejos, C. H. Lewenkopf, and E. R. Mucciolo, Phys. Rev. B 60, 13682 (1999).

[16] E. E. Narimanov, N. R. Cerruti, H. U. Baranger, and S. Tomsovic, Phys. Rev. Lett. 83, 2640 (1999).

[17] L. Kaplan, Phys. Rev. E 62, 3476 (2000), [arXiv:nlin.CD/0003013].

[18] E. E. Narimanov, H. U. Baranger, N. R. Cerruti, and S. Tomsovic, submitted to Phys. Rev. B, arXiv:condmat/0101034 (unpublished).

[19] S. R. Patel, D. R. Stewart, C. M. Marcus, M. Gokcedag, Y. Alhassid, A. D. Stone, C. I. Duruos, and J. J. S. Harris, Phys. Rev. Lett. 81, 5900 (1998).

[20] A. M. Chang, H. U. Baranger, L. N. Pfeiffer, K. W. West, and T. Y. Chang, Phys. Rev. Lett. 76, 1695 (1996).

[21] J. A. Folk, S. R. Patel, S. F. Godijn, A. G. Huibers, S. M. Cronenwett, and C. M. Marcus, Phys. Rev. Lett. 76, 1699 (1996).

[22] M. G. Mayer, Phys. Rev. 60, 184 (1941).

[23] R. Latter, Phys. Rev. 99, 510 (1955).

[24] O. Bohigas, X. Campi, H. Krivine, and J. Treiner, Phys. Lett. 64, 381 (1976).

[25] U. Sivan, R. Berkovits, Y. Aloni, O. Prus, A. Auerbach, and G. Ben-Yoseph, Phys. Rev. Lett. 77, 1123 (1996).

[26] F. Simmel, T. Heinzel, and D. A. Wharam, Europhys. Lett. 38, 123 (1997).

[27] S. R. Patel, S. M. Cronenwett, D. R. Stewart, A. G. Huibers, C. M. Marcus, C. I. Duruz, J. S. Harris, K. Campman, and A. C. Gossard, Phys. Rev. Lett. 80, 4522 (1998).

[28] F. Simmel, D. Abusch-Magder, D. A. Wharam, M. A. Kastner, and J. P. Kotthaus, Phys. Rev. B 59, R10441 (1999).
[29] S. Lüscher, T. Heinzel, K. Ensslin, W. Wegscheider, and M. Bichler, arXiv:cond-mat/0002226 (unpublished).

[30] T. T. Ong, H. U. Baranger, C. M. Marcus, and S. R. Patel (unpublished).

[31] R. Berkovits, Phys. Rev. Lett. 81, 2128 (1998).

[32] P. Walker, G. Montambaux, and Y. Gefen, Phys. Rev. Lett. 82, 5329 (1999).

[33] P. Walker, G. Montambaux, and Y. Gefen, Phys. Rev. B 60, 2541 (1999).

[34] A. Cohen, K. Richter, and R. Berkovits, Phys. Rev. B 60, 2536 (1999).

[35] K.-H. Ahn, K. Richter, and I.-H. Lee, Phys. Rev. Lett. 83, 4144 (1999).

[36] N. W. Ashcroft and N. D. Mermin, Solid State Physics (Holt, Rinehart and Winston, New York, 1976), p. 344.

[37] M. Koskinen, M. Manninen, and S. M. Reimann, Phys. Rev. Lett. 79, 1389 (1997).

[38] I.-H. Lee, V. Rao, R. M. Martin, and J.-P. Leburton, Phys. Rev. B 57, 9035 (1998).

[39] K. Hirose and N. S. Wingreen, Phys. Rev. B 59, 4604 (1999).

[40] O. Prus, A. Auerbach, Y. Aloni, U. Sivan, and R. Berkovits, Phys. Rev. B 54, R14289 (1996).

[41] R. Berkovits and B. Altshuler, Phys. Rev. B 55, 5297 (1997).

[42] Y. M. Blanter, A. D. Mirlin, and B. A. Muzykantskii, Phys. Rev. Lett. 78, 2449 (1997).

[43] P. W. Brouwer, Y. Oreg, and B. I. Halperin, Phys. Rev. B 60, R13977 (1999).

[44] H. U. Baranger, D. Ullmo, and L. I. Glazman, Phys. Rev. B 61, R2425 (2000).

[45] I. L. Kurland, I. L. Aleiner, and B. L. Altshuler, Phys. Rev. B 62, 14886 (2000).

[46] P. Jacquod and A. D. Stone, Phys. Rev. Lett. 84, 3938 (2000).

[47] P. Jacquod and A. D. Stone, cond-mat/0102029 (unpublished).

[48] M. Gutzwiller, Chaos in Classical and Quantum Mechanics (Spring-Verlag, New York, 1991).

[49] B. L. Altshuler and B. D. Simons, in Mesoscopic Quantum Physics, edited by E. Akkermans, G. Montambaux, J.-L. Pichard, and J. Zinn-Justin (Elsevier Science, Amsterdam, 1995), pp. 1-98.

[50] V. M. Strutinskii, Nucl. Phys A 122, 1 (1968).

[51] M. Brack, J. Damgøard, A. S. Jensen, H. C. Pauli, and V. M. Strutinsky, Rev. Mod. Phys. 44, 320 (1972).

[52] D. Ullmo, T. Nagano, S. Tomsovic, and H. U. Baranger, arXiv:cond-mat/0007330 (unpublished).

[53] Chaos and Quantum Physics, edited by M.-J. Giannoni, A. Voros, and J. Zinn-Justin (North-Holland, New York, 1991).

[54] M. V. Berry, J. Phys. A 10, 2083 (1977).

[55] M. Srednicki, Phys. Rev. E 54, 954 (1996).

[56] O. Agam, N. S. Wingreen, B. L. Altshuler, D. C. Ralph, and M. Tinkham, Phys. Rev. Lett. 78, 1956 (1997).

[57] I. L. Aleiner and L. I. Glazman, Phys. Rev. B 57, 9608 (1998).

[58] Y. M. Blanter, Phys. Rev. B 54, 12807 (1996).

[59] Y. M. Blanter and A. D. Mirlin, Phys. Rev. B 55, 6514 (1997).

[60] P. Leboeuf and A. Monastra, Phys. Rev. B 62, 12617 
(2000).

[61] M. Wilkinson, J. Phys. A 22, 2795 (1989).

[62] E. J. Austin and M. Wilkinson, Nonlinearity 5, 1137 (1992).

[63] Y. V. Fyodorov and A. D. Mirlin, Phys. Rev. Lett. 67,
2405 (1996).

[64] T. Heinzel, private communication (unpublished).

[65] D. R. Stewart, D. Sprinzak, C. M. Marcus, C. I. Duruoz, and J. S. Harris, Science 278, 1784 (1997). 